\title{
Fokker-Planck Equations for SPDE with Non-trace-class Noise
}

\author{
G. Da Prato • F. Flandoli • M. Röckner
}

Received: 28 June 2013 / Accepted: 19 August 2013 / Published online: 10 September 2013

(C) School of Mathematical Sciences, University of Science and Technology of China and Springer-Verlag Berlin Heidelberg 2013

\begin{abstract}
In this paper we develop a new technique to prove existence of solutions of Fokker-Planck equations on Hilbert spaces for Kolmogorov operators with nontrace-class second order coefficients or equivalently with an associated stochastic partial differential equation (SPDE) with non-trace-class noise. Applications include stochastic 2D and 3D-Navier-Stokes equations with non-trace-class additive noise.
\end{abstract}

Keywords Fokker-Planck equation · Kolmogorov operator - Stochastic Partial Differential Equations · Stochastic Navier-Stokes equations · Non-trace-class noise

Mathematics Subject Classification (2010) 35Q84 · 60H15 · 35R60 - 82C31 . 35Q30 $\cdot 76 \mathrm{D} 05 \cdot 76 \mathrm{M} 35$

\section{Introduction}

Our aim is to solve the infinite dimensional Fokker-Planck Equation

$$
L^{*} \mu=0,\left.\quad \mu\right|_{t=0}=\mu_{0}
$$

in a space of measure valued solutions of the form $\mu_{t} d t$ where $\mu_{t}$ are probability measures on $H$. The problem has been studied intensively in recent years (see e.g.,

G. Da Prato

Scuola Normale Superiore, Pisa, Italy

F. Flandoli

Università di Pisa, Pisa, Italy

M. Röckner ( $\square)$

University of Bielefeld, Bielefeld, Germany

e-mail: roeckner@math.uni-bielefeld.de 
$[4-6,8,20]$ and the references therein). Concerning existence there are two different approaches to infinite dimensional Fokker-Planck equations depending on whether the second order coefficient of the corresponding Kolmogorov operator $L$ (see below) is of trace class or not. The first case is studied in detail in [8] (including also the case of continuity equations whose second order coefficient is identically zero) and the approach is based on the method of Lyapunov functions. This method, however, so far could not be implemented when the second order coefficient is not of trace class. This case has been studied in [5] and [20], using an approximation technique, based on solving the stochastic differential equations associated to the approximating Kolmogorov operator $L_{n}$. Then suitable accumulation points are proved to be solutions of the limiting given Fokker-Planck equation. The results in [5] and [20] are, however, very limited in applications, e.g., essentially only Fokker-Planck equations associated to stochastic reaction-diffusion and Burgers equations with non-trace-class noise (including white noise) or combinations of such are covered.

In this paper we develop a new general approach for this "non-trace-class case" which applies to a much wide class of examples, including in particular, the FokkerPlanck equation of the stochastic 2D and 3D Navier-Stokes equations with non-traceclass noise (see Sect. 5.2 below). For the sake of simplicity we restrict ourselves to the case where the second coefficient of $L$ is constant. In the language of stochastic equations we restrict ourselves to additive noise.

The Kolmogorov operator $L$ in equation $(F P E)$ above is defined as

$$
(L u)(x, t):=\frac{\partial u}{\partial t}(x, t)+\sum_{i=1}^{\infty} a^{i}\left(\partial_{x_{i}}^{2} u\right)(x, t)+\sum_{i=1}^{\infty} b^{i}(x, t)\left(\partial_{x_{i}} u\right)(x, t)
$$

on all functions $u: H \times[0, T] \rightarrow \mathbb{R}$ which are smooth and finite dimensional (also called cylindrical). Here $H$ is a separable Hilbert space (norm $\|.\|_{H}$, inner product $\left.\langle., .\rangle_{H}\right),\left(e_{n}\right)$ is a c.o.s. in $H, H_{n}$ is the span of $e_{1}, \ldots, e_{n}, \pi_{n}$ the corresponding finite dimensional projection, and the attribute "finite dimensional" to $u$ means that $u(t, x)=u\left(\pi_{n} x, t\right)$ for all $x$, for some $n \in \mathbb{N}$ and $u_{n}: \mathbb{R}^{n} \times[0, T] \rightarrow \mathbb{R}$. Here and below we shall always identify $H_{n}$ with $\mathbb{R}^{n}$ fixing $\left(e_{n}\right)$.

The ideas of the present work are general, in particular the approach by an auxiliary Fokker-Planck Equation on a product space. We develop them under quite general assumptions which include basic cases like stochastic semilinear parabolic equations with linear growth (but see also Remark 14) and, mainly, stochastic 2D and 3D Navier-Stokes equations. A direct solution of the Fokker-Planck equation corresponding to these equations when the noise has the covariance of the class considered here (not trace class or as general as possible) is new; for other approaches to the existence of solutions for the stochastic 2D and 3D Navier-Stokes equations, especially in the direction of general covariance, see for instance $[1,2,10,13]$ in $2 \mathrm{D}$ and $[11,14-18]$ in $3 \mathrm{D}$.

\section{Assumptions and Main Result}

The numbers $a^{i} \geq 0$ and the measurable functions $b^{i}: H \times[0, T] \rightarrow \mathbb{R}$ are subject to a series of assumptions. We do not assume the finite trace condition $\sum_{i=1}^{\infty} a^{i}<\infty$ as 
in $[4,8]$, but we do not allow dependence on $(x, t)$ (hence our applications restrict to additive noise).

Let $E \subset H$ be a separable Banach space with dense continuous injection. Denote the norm in $E$ by $\|.\|_{E}$. We assume $e_{i} \in E$ and other conditions below.

We assume that the $b^{i}$ have the structure

$$
b^{i}(x, t)=-\alpha_{i}^{2} x_{i}+f^{i}(x, t)
$$

with real numbers $\alpha_{i}^{2}>0$ and continuous functions $f^{i}: H \times[0, T] \rightarrow \mathbb{R}$. The basic assumption on the sequences $\left(a^{i}\right),\left(\alpha_{i}^{2}\right)$ is

$$
\lim _{i \rightarrow \infty} \alpha_{i}^{2}=\infty, \quad \sum_{i=1}^{\infty} \frac{a^{i}}{\alpha_{i}^{2}}<\infty .
$$

Concerning the sequence of functions $f^{i}(x, t)$, we assume

$$
\begin{gathered}
\left|f^{i}(x, t)\right| \leq C_{i}\left(1+\|x\|_{H}^{p_{0}}\right), \quad f^{i}(\cdot, t) \text { locally Lipschitz in } x, \\
\text { uniformly in } t \in[0, T]
\end{gathered}
$$

for some $C_{i}>0, p_{0} \geq 1$.

Denote by $V$ the Hilbert space

$$
V=\left\{x \in H:\|x\|_{V}^{2}:=\sum_{i=1}^{\infty} \alpha_{i}^{2} x_{i}^{2}<\infty\right\}
$$

where $x_{i}=\left\langle x, e_{i}\right\rangle_{H}$. It is compactly embedded in $H$, by assumption (2.1). Let $V^{\prime}$ be the dual space of $V$ and let us use the identification $H=H^{\prime}$, so that $V \subset H \subset V^{\prime}$, with dense injections. We write $\langle.,$.$\rangle for the dual pairing between$ $V^{\prime}$ and $V$, so $\langle x, y\rangle=\langle x, y\rangle_{H}$ when $x \in H, y \in V$. Note that then $\pi_{n}$ has a natural extension from $H$ to $V^{\prime}$. We assume also that the Banach space $E \cap V$ is dense in $H$.

We assume that there is a Borel function $f: E \times[0, T] \rightarrow V^{\prime}$ such that $f^{i}(x, t)=$ $\left\langle f(x, t), e_{i}\right\rangle$ (in other words, we assume that the series $f(x, t):=\sum_{i=1}^{\infty} f^{i}(x, t) e_{i}$ converges in $V^{\prime}$ for all $x \in E$ and $\left.t \in[0, T]\right)$, and on $f$ we assume for some $C$, $k_{0} \in(0, \infty)$ and $\eta \in(0,1)$

$$
\langle f(v+z, t), v\rangle \leq \eta\|v\|_{V}^{2}+C\|v\|_{H}^{2}\left(\|z\|_{E}^{2}+1\right)+C\|z\|_{E}^{k_{0}}+C
$$

for all $z \in E, v \in E \cap V$. When $f$ grows more than linearly, this assumption embodies a form of cancellation.

Let $\left(\beta_{i}(t)\right)_{t \geq 0}$ be a sequence of independent Wiener processes on a probability space $(\Omega, \mathcal{F}, P)$ with normal filtration $\left(\mathcal{F}_{t}\right)_{t \geq 0}$. Then the series of stochastic integrals, parametrized by $\lambda \geq 0$,

$$
Z_{t}^{\lambda}:=\sum_{i=1}^{\infty} \int_{0}^{t} e^{-(t-s)\left(\alpha_{i}^{2}+\lambda\right)} \sqrt{a^{i}} d \beta_{i}(s) e_{i}
$$


defines a continuous Gaussian process in $H$, by assumption (2.1). Our last assumption on $E$ is that

$$
Z^{\lambda} \text { is an } L^{2 \vee k_{0}}(0, T ; E) \text {-valued Gaussian variable }
$$

and for every $r>0$ one has

$$
\lim _{\lambda \rightarrow \infty} P\left(\int_{0}^{T}\left\|Z_{t}^{\lambda}\right\|_{E}^{2} d t>r^{2}\right)=0 .
$$

Let $(A, D(A))$ and $(Q, D(Q))$ denote the self-adjoint linear operators

$$
\begin{array}{ll}
D(A)=\left\{x \in H: \sum_{i=1}^{\infty}\left(\alpha_{i}^{2}\left\langle x, e_{i}\right\rangle_{H}\right)^{2}<\infty\right\}, & A x=-\sum_{i=1}^{\infty} \alpha_{i}^{2}\left\langle x, e_{i}\right\rangle_{H} e_{i}, \\
D(Q)=\left\{x \in H: \sum_{i=1}^{\infty}\left(a^{i}\left\langle x, e_{i}\right\rangle_{H}\right)^{2}<\infty\right\}, & Q x=\sum_{i=1}^{\infty} a^{i}\left\langle x, e_{i}\right\rangle_{H} e_{i} .
\end{array}
$$

Then $Z_{t}^{\lambda}, t \geq 0$, defined in (2.4), can be rewritten as

$$
Z_{t}^{\lambda}=\int_{0}^{t} e^{(t-s)(A-\lambda)} \sqrt{Q} d W_{s}, \quad t \geq 0,
$$

which is a continuous Gaussian process in $H$, with trace-class covariance (by assumption (2.1))

$$
Q_{t}^{\lambda}=\int_{0}^{t} e^{s(A-\lambda)} Q e^{s(A-\lambda)} d s, \quad t \geq 0 .
$$

By assumption $(2.5), Z_{\text {. }}^{\lambda}$ is an $L^{2}(0, T ; E)$-valued Gaussian variable and solves the linear stochastic equation in $H$

$$
d Z_{t}^{\lambda}=A Z_{t}^{\lambda} d t+\sqrt{Q} d W_{t}-\lambda Z_{t} d t, \quad Z_{0}^{\lambda}=0 .
$$

Remark 1 Under a natural condition on $e^{t A}, t \geq 0$, (2.5) with $Z_{t}^{0}$ replacing $Z_{t}^{\lambda}$ actually implies (2.6). Indeed, under the assumption that the restriction of $e^{t A}, t \geq 0$, is a strongly continuous semigroup on $E,(2.6)$ holds. This can be proved as follows: fix $i \in \mathbb{N}$. Then by Itô's product rule

$$
e^{\lambda t} \int_{0}^{t} e^{\alpha_{i}^{2} s} d \beta_{i}(s)=\int_{0}^{t} e^{\lambda s} e^{\alpha_{i}^{2} s} d \beta_{i}(s)+\int_{0}^{t} \int_{0}^{s} e^{\alpha_{i}^{2} r} d \beta_{i}(r) \lambda e^{\lambda s} d s .
$$

Hence

$$
\begin{aligned}
\int_{0}^{t} e^{-(t-s)\left(\alpha_{i}^{2}+\lambda\right)} d \beta_{i}(s)= & \int_{0}^{t} e^{-(t-s) \alpha_{i}^{2}} d \beta_{i}(s) \\
& -\int_{0}^{t} \int_{0}^{s} e^{-(t-r) \alpha_{i}^{2}} d \beta_{i}(r) \lambda e^{-\lambda(t-s)} d s .
\end{aligned}
$$


Multiplying by $\sqrt{a^{i}} e_{i}$ and taking summation over $i \in \mathbb{N}$ (here the convergence holds in $H$ ), we obtain

$$
\begin{aligned}
Z_{t}^{\lambda} & =\int_{0}^{t} e^{(t-s) A} \sqrt{Q} d W_{t}-\int_{0}^{t} e^{(t-s) A} \int_{0}^{s} e^{(s-r) A} \sqrt{Q} d W_{r} \lambda e^{-\lambda(t-s)} d s \\
& =Z_{t}^{0}-\left(1-e^{-\lambda t}\right) \int_{0}^{t} e^{(t-s) A} Z_{s}^{0} \rho_{\lambda}(t-s) d s,
\end{aligned}
$$

where $\rho_{\lambda}(t-s)=\mathbb{1}_{[0, t]}(s)\left(1-e^{-\lambda t}\right)^{-1} \lambda e^{-\lambda(t-s)}$ weakly converges to the Dirac measure in $t$ as $\lambda \rightarrow \infty$.

Hence, since $Z_{t}^{0} \in E$ for $d t$-a.e. $t \in[0, T]$, it follows that

$$
\lim _{\lambda \rightarrow \infty} \int_{0}^{T}\left\|Z_{t}^{\lambda}\right\|_{E}^{2} d t=0 \quad P \text {-a.s. }
$$

in particular (2.6) holds.

More precisely,

$$
\begin{aligned}
& \left(\int_{0}^{T}\left\|Z_{t}^{0}-\int_{0}^{t} e^{(t-s) A} Z_{s}^{0} \rho_{\lambda}(t-s) d s\right\|_{E}^{2} d t\right)^{1 / 2} \\
& \quad=\left(\int_{0}^{T}\left\|\int_{0}^{t}\left(Z_{t}^{0}-e^{(t-s) A} Z_{s}^{0}\right) \rho_{\lambda}(t-s) d s\right\|_{E}^{2} d t\right)^{1 / 2} \\
& \leq\left(\int_{0}^{T} T \int_{0}^{t}\left\|Z_{t}^{0}-e^{s A} Z_{t-s}^{0}\right\|_{E}^{2} \rho_{\lambda}(s) d s d t\right)^{1 / 2} \\
& \quad \leq T^{1 / 2}\left[\int_{0}^{T} \int_{0}^{T}\left\|Z_{t}^{0}-Z_{t-s}^{0}\right\|_{E}^{2} d t \rho_{\lambda}(s) d s\right]^{1 / 2} \\
& \quad+T^{1 / 2}\left[\int_{0}^{T} \int_{0}^{T-s}\left\|\left(1-e^{s A}\right) Z_{t}^{0}\right\|_{E}^{2} d t \rho_{\lambda}(s) d s\right]^{1 / 2} .
\end{aligned}
$$

Since for $P$-a.e. $\omega \in \Omega$ the first inner integral is continuous and bounded in $s \in[0, T]$, this implies (2.7).

Remark 2 The limit property in assumption (2.6) is needed because of the power 2 of $\|z\|_{E}^{2}$ in the term $C\|v\|_{H}^{2}\left(\|z\|_{E}^{2}+1\right)$ of assumption (2.3), which is a sort of critical value case; if instead we took the term $C\|v\|_{H}^{2}\left(\|z\|_{E}^{\beta}+1\right)$ with $\beta<2$ in (2.3), we only need $Z_{\text {. }}^{\lambda}$ being $L^{2}(0, T ; E)$-valued, but this is too restrictive for applications to Navier-Stokes equations.

We define $D(L)$ to be the linear space of finite dimensional regular functions $u$ : $H \times[0, T] \rightarrow \mathbb{R}$, i.e., $u(t, x)=u_{N}\left(\left\langle e_{1}, x\right\rangle, \ldots,\left\langle e_{N}, x\right\rangle, t\right)$ such that $u_{N} \in C_{b}^{2,1}\left(\mathbb{R}^{N} \times\right.$ $[0, T])$ and $u(x, T)=0 . D(L)$ is then a (point and measure) separating class. 
Definition 3 A family of Borel probability measures $\left(\mu_{t}(d x)\right)_{t \in[0, T]}$ on $H$, measurable in $t$, is a solution of the Fokker-Planck equation (FPE) above if

$$
\int_{0}^{T} \int_{H}\|x\|_{H}^{p_{0}} \mu_{t}(d x) d t<\infty
$$

where $p_{0}$ is given in assumption (2.2), and

$$
\int_{0}^{T} \int_{H}(L u)(x, t) \mu_{t}(d x) d t+\int_{H} u(x, 0) \mu_{0}(d x)=0
$$

for all $u \in D(L)$.

The double integral in the above formulation is then well defined (see Remark 6 below) because of (2.2) and the assumed moment condition. We can now state our main theorem.

Theorem 4 Under the assumptions (2.1)-(2.6), for any Borel probability measure $\mu_{0}$ on $H$ such that

$$
\int_{H}\|x\|_{H}^{p_{1}} \mu_{0}(d x)<\infty
$$

for some $p_{1}>p_{0}$, equation (FPE) has a solution.

In Sect. 5 we shall give two examples: the case of measurable drift of at most linear growth and the 2D and 3D Navier-Stokes equations.

\section{Auxiliary Fokker-Planck Equation on Product Space}

On finite dimensional regular functions $\tilde{u}(v, z, t), \tilde{u}: H \times H \times[0, T] \rightarrow \mathbb{R}$, more precisely for $\tilde{u} \in D(\widetilde{L})$, where $D(\widetilde{L})$ is defined analogously to $D(L)$ with $H \times H$ replacing $H$, define the auxiliary Kolmogorov operator

$$
\begin{aligned}
(\widetilde{L} \widetilde{u})(v, z, t):= & \frac{\partial \widetilde{u}}{\partial t}(v, z, t)+\sum_{i=1}^{\infty}\left(\left(a^{i} \partial_{z_{i}}^{2}-\left(\alpha_{i}^{2}+\lambda\right) z_{i} \partial_{z_{i}}\right) \widetilde{u}\right)(v, z, t) \\
& +\sum_{i=1}^{\infty}\left(-\alpha_{i}^{2} v_{i}+f^{i}(v+z, t)+\lambda z_{i}\right)\left(\partial_{v_{i}} \widetilde{u}\right)(v, z, t)
\end{aligned}
$$

Here $\lambda \geq 0$ is a parameter. In the simplest cases (application to drifts of at most linear growth, for instance) we could simply take $\lambda=0$, but for some applications we need suitable values of $\lambda$. The trick of this parameter has been introduced in [9] to prove the existence of random attractors for the Navier-Stokes equations, and here it will be used to prove moment estimates for solutions. 
Definition 5 A family of Borel probability measures $\left(\tilde{\mu}_{t}(d v, d z)\right)_{t \in[0, T]}$ on $H \times H$, measurable in $t$, is a solution of the Fokker-Planck equation on product space

$$
\widetilde{L}^{*} \tilde{\mu}=0,\left.\quad \tilde{\mu}\right|_{t=0}=\tilde{\mu}_{0},
$$

if

$$
\int_{0}^{T} \int_{H \times H}\left(\|v\|_{H}^{p_{0}}+\|z\|_{H}^{p_{0}}\right) \widetilde{\mu}_{t}(d v, d z) d t<\infty,
$$

where $p_{0}$ is given in assumption (2.2), and

$$
\int_{0}^{T} \int_{H \times H}(\widetilde{L} \widetilde{u})(v, z, t) \tilde{\mu}_{t}(d v, d z) d t+\int_{H \times H} \widetilde{u}(v, z, 0) \tilde{\mu}_{0}(d v, d z)=0
$$

for all $\tilde{u} \in D(\widetilde{L})$.

Remark 6 The double integral in the above formulation is well defined. Indeed, when $\widetilde{u} \in D(\widetilde{L})$, the term $\sum_{i=1}^{\infty}\left(\left(a^{i} \partial_{z_{i}}^{2}-\left(\alpha_{i}^{2}+\lambda\right) z_{i} \partial_{z_{i}}\right) \widetilde{u}\right)(v, z, t)$ reduces to a finite sum and we have the bound

$$
\left|\sum_{i=1}^{\infty}\left(\left(a^{i} \partial_{z_{i}}^{2}-\left(\alpha_{i}^{2}+\lambda\right) z_{i} \partial_{z_{i}}\right) \widetilde{u}\right)(v, z, t)\right| \leq C+C\|z\|_{H}
$$

which is integrable with respect to $\widetilde{\mu}_{t}(d v, d z) d t$ by the integrability assumption of the definition. Similarly, the term

$$
\sum_{i=1}^{\infty}\left(-\alpha_{i}^{2} v_{i}+f^{i}(v+z, t)+\lambda z_{i}\right)\left(\partial_{v_{i}} \tilde{u}\right)(v, z, t)
$$

reduces to a finite sum and we have the bound

$$
\begin{aligned}
& \left|\sum_{i=1}^{\infty}\left(-\alpha_{i}^{2} v_{i}+f^{i}(v+z, t)+\lambda z_{i}\right)\left(\partial_{v_{i}} \widetilde{u}\right)(v, z, t)\right| \\
& \quad \leq C\|v\|_{H}+C \sum_{i=1}^{N}\left|f^{i}(v+z, t)\right|+C\|z\|_{H}
\end{aligned}
$$

for some $N>0$, and thus by our main assumptions this is dominated by

$$
C\|v\|_{H}+C_{N, p}\left(1+\|v\|_{H}^{p_{0}}+\|z\|_{H}^{p_{0}}\right)+C\|z\|_{H},
$$

which is again integrable with respect to $\widetilde{\mu}_{t}(d v, d z) d t$.

Remark 7 Let $\pi_{i}: H \times H \rightarrow H, i=1,2$, denote the canonical projections and fix $t \in[0, T]$. Then $\tilde{\mu}_{t} \circ \pi_{2}^{-1}=$ the law of $Z_{t}^{\lambda}=N_{Q_{t}}$, i.e., the mean zero Gaussian measure on $H$ with covariance operator $Q_{t}$, where

$$
Q_{t}:=\int_{0}^{t} e^{-2 s(A+\lambda)} Q d s .
$$


Indeed, it is easy to check that $\left(\tilde{\mu}_{t} \circ \pi_{2}^{-1}\right)(d x) d t$ solves $(F P E)$ with $L$ replaced by

$$
L_{0} u:=\frac{\partial u}{\partial t}+\sum_{i=1}^{\infty}\left(a^{i} \partial_{z_{i}}^{2}-\left(\alpha_{i}^{2}+\lambda\right) z_{i}\right) \partial_{z_{i}} u,
$$

with domain $D(L)$ (as above) and $\mu_{0}=\delta_{0}$. But for this Fokker-Planck equation $\left(N_{Q_{t}}\right)_{t \in[0, T]}$ is the unique solution.

Remark 8 In Definition 5 it is sufficient to assume

$$
\int_{0}^{T} \int_{H \times H}\left(\|v+z\|_{H}^{p_{0}}+\|v\|_{H}+\|z\|_{H}\right) \tilde{\mu}_{t}(d v, d z) d t<\infty .
$$

Lemma 9 If $\tilde{\mu}_{t}(d v, d z) d t$ is a solution of $(\widetilde{F P E})$ on product space and if $\tilde{\mu}_{0}$ and $\mu_{0}$ are related by the condition

$$
\int_{H \times H} \varphi(v+z) \tilde{\mu}_{0}(d v, d z)=\int_{H} \varphi(x) \mu_{0}(d x)
$$

then $\mu_{t}(d x)$, defined for all $t \in[0, T]$ as

$$
\int_{H} \varphi(x) \mu_{t}(d x):=\int_{H \times H} \varphi(v+z) \tilde{\mu}_{t}(d v, d z), \quad \varphi \in C_{b}(H),
$$

is a weak solution of (FPE).

Proof Step 1. Let $u \in D(L)$. Define

$$
\tilde{u}(v, z, t):=u(v+z, t) .
$$

Then we have

$$
\begin{gathered}
(L u)(v+z, t)=\frac{\partial u}{\partial t}(v+z, t)+\sum_{i=1}^{\infty} a^{i}\left(\partial_{x_{i}}^{2} u\right)(v+z, t) \\
+\sum_{i=1}^{\infty} b^{i}(v+z, t)\left(\partial_{x_{i}} u\right)(v+z, t) \\
(\widetilde{L} \widetilde{u})(v, z, t)=\frac{\partial u}{\partial t}(v+z, t)+\sum_{i=1}^{\infty}\left(\left(a^{i} \partial_{x_{i}}^{2}-\left(\alpha_{i}^{2}+\lambda\right) z_{i} \partial_{x_{i}}\right) u\right)(v+z, t) \\
+\sum_{i=1}^{\infty}\left(-\alpha_{i}^{2} v_{i}+f^{i}(v+z, t)+\lambda z_{i}\right)\left(\partial_{x_{i}} u\right)(v+z, t) \\
=\frac{\partial u}{\partial t}(v+z, t)+\sum_{i=1}^{\infty} a^{i}\left(\partial_{x_{i}}^{2} u\right)(v+z, t) \\
+\sum_{i=1}^{\infty}\left(-\alpha_{i}^{2}\left(v_{i}+z_{i}\right)+f^{i}(v+z, t)\right)\left(\partial_{x_{i}} u\right)(v+z, t)
\end{gathered}
$$


So, we deduce

$$
(L u)(v+z, t)=(\widetilde{L} \widetilde{u})(v, z, t) .
$$

Step 2. The integrability condition of Definition 3 holds, since by definition of $\mu_{t}(d x)$, we have

$$
\begin{aligned}
\int_{0}^{T} \int_{H}\|x\|_{H}^{p_{0}} \mu_{t}(d x) d t & =\int_{0}^{T} \int_{H \times H}\|v+z\|_{H}^{p_{0}} \tilde{\mu}_{t}(d v, d z) d t \\
& \leq C_{p_{0}} \int_{0}^{T} \int_{H \times H}\left(\|v\|_{H}^{p_{0}}+\|z\|_{H}^{p_{0}}\right) \tilde{\mu}_{t}(d v, d z) d t<\infty .
\end{aligned}
$$

Step 3. By definition of $\mu_{t}(d x)$, we have

$$
\int_{0}^{T} \int_{H}(L u)(x, t) \mu_{t}(d x) d t=\int_{0}^{T} \int_{H \times H}(L u)(v+z, t) \tilde{\mu}_{t}(d v, d z) d t .
$$

Hence, by the previous step, with $\tilde{u}(v, z, t):=u(v+z, t)$,

$$
\int_{0}^{T} \int_{H}(L u)(x, t) \mu_{t}(d x) d t=\int_{0}^{T} \int_{H \times H}(\tilde{L} \tilde{u})(v, z, t) \tilde{\mu}_{t}(d v, d z) d t .
$$

This and (3.1) imply the claim of the lemma.

Remark 10 For a given Borel probability measure $\mu_{0}$ on $H$ it is easy to find a Borel probability measure $\tilde{\mu}_{0}$ on $H \times H$ such that (3.1) holds. Simply, define

$$
\tilde{\mu}_{0}(d v, d z):=\varepsilon_{(0, x)}(d v, d z) \mu_{0}(d x)
$$

where $\varepsilon_{(0, x)}$ is the Dirac measure in $(0, x) \in H \times H$. Then clearly (3.1) holds. Then the second marginal of $\tilde{\mu}_{0}$ is just $\mu_{0}$. Another choice with first marginal equal to $\mu_{0}$ is $\tilde{\mu}_{0}=\mu_{0} \otimes \delta_{0}$. Hence any convex combination of these two satisfies (3.1).

Thus, to prove existence of solutions of $(F P E)$, it is sufficient to solve the auxiliary Fokker-Planck equation $(\widetilde{F P E})$, with suitable initial condition.

\section{Existence Theorem for the Auxiliary Equation $(\widetilde{F P E})$}

In this section we want to prove the existence of a solution to the equation (called above $(\widetilde{F P E}))$

$$
\int_{0}^{T} \int_{H \times H}(\widetilde{L} \tilde{u})(v, z, t) \tilde{\mu}_{t}(d v, d z) d t+\int_{H \times H} \tilde{u}(v, z, 0) \tilde{\mu}_{0}(d v, d z)=0
$$

with the initial condition $\tilde{\mu}_{0}=\mu_{0} \otimes \delta_{0}$. This initial condition satisfies (3.1). One can decompose $\tilde{\mu}_{0}$ in other ways (see Remark 10). 
Theorem 11 Let the assumptions (2.1)-(2.6) hold and let $\mu_{0}$ be a Borel probability measure on $H$ such that

$$
\int_{H}\|x\|_{H}^{p_{1}} \mu_{0}(d x)<\infty
$$

for some $p_{1}>p_{0}$. Then there exists $\lambda_{0} \geq 0$ such that for every $\lambda \geq \lambda_{0}$ equation $(\widetilde{F P E})$ has a solution.

The proof is given in the following subsections. By Lemma 9, this proves our main Theorem 4.

\subsection{A Consequence of Fernique's Theorem}

Proposition 12 For every $K>0$ there is $\lambda_{0}>0$ such that for all $\lambda \geq \lambda_{0}$

$$
E\left[e^{\int_{0}^{T} K\left\|Z_{t}^{\lambda}\right\|_{E}^{2} d t}\right] \leq e^{\frac{1}{4}}+\frac{e^{2}}{e^{2}-1} .
$$

Proof Since $Z_{\text {. }}^{\lambda}$ is a Gaussian r.v. in the Banach space $L^{2}(0, T ; E)$, Fernique's theorem states that there exists $\gamma_{0}>0$ such that

$$
E\left[e^{\gamma \int_{0}^{T}\left\|Z_{t}^{\lambda}\right\|_{E}^{2} d t}\right]<\infty
$$

for all $\gamma \in\left(0, \gamma_{0}\right)$. We need a relation between $\lambda$ and $\gamma_{0}$, so we use the following version of Fernique's theorem (see [12]): given an $L^{2}(0, T ; E)$-valued Gaussian variable $Z$, if two real numbers $\gamma, r>0$ satisfy

$$
\log \left(\frac{1-P\left(\|Z\|_{L^{2}(0, T ; E)} \leq r\right)}{P\left(\|Z\|_{L^{2}(0, T ; E)} \leq r\right)}\right)+32 \gamma r^{2} \leq-1,
$$

then

$$
E\left[e^{\gamma\|Z\|_{L^{2}(0, T ; E)}^{2}}\right] \leq e^{16 \gamma r^{2}}+\frac{e^{2}}{e^{2}-1} .
$$

Now, given $K>0$, choose $r=\frac{1}{8 \sqrt{K}}$. By assumption (2.6), there exists $\lambda_{0}>0$ such that

$$
P\left(\int_{0}^{T}\left\|Z_{t}^{\lambda}\right\|_{E}^{2} d t \leq r\right) \geq \frac{1}{e^{-3 / 2}+1}, \quad \forall \lambda \geq \lambda_{0} .
$$

Then

$$
\log \left(\frac{1-P\left(\int_{0}^{T}\left\|Z_{t}^{\lambda}\right\|_{E}^{2} d t \leq r\right)}{P\left(\int_{0}^{T}\left\|Z_{t}^{\lambda}\right\|_{E}^{2} d t \leq r\right)}\right) \leq-\frac{3}{2}
$$

Therefore,

$$
\log \left(\frac{1-P\left(\left\|Z^{\lambda}\right\|_{L^{2}(0, T ; E)} \leq r\right)}{P\left(\left\|Z^{\lambda}\right\|_{L^{2}(0, T ; E)} \leq r\right)}\right)+32 K r^{2} \leq-1
$$


By the previous version of Fernique's theorem we have

$$
E\left[e^{K\left\|Z_{.}^{\lambda}\right\|_{L^{2}(0, T ; E)}^{2}}\right] \leq e^{\frac{1}{4}}+\frac{e^{2}}{e^{2}-1} .
$$

The proof is complete.

\subsection{Approximating Problem, Moment Estimate}

We use the same notations and objects of the previous subsection but we enlarge, if necessary, the filtered probability space $\left(\Omega, \mathcal{F},\left(\mathcal{F}_{t}\right)_{t \geq 0}, P\right)$ in such a way that there exists an $\mathcal{F}_{0}$-measurable r.v. $V(0)$ with law $\mu_{0}$. Set

$$
A_{n} x=-\sum_{i=1}^{n} \alpha_{i}^{2}\left\langle x, e_{i}\right\rangle_{H} e_{i}, \quad f_{n}(x, t)=\sum_{i=1}^{n} f^{i}(x, t) e_{i}=\pi_{n} f(x, t)
$$

(the latter equality is true only for $x \in E$ ). Consider the finite dimensional system in $\pi_{n}(H)$ for the unknown $V_{n}(t)$, driven by the known Gaussian process $Z_{t}^{\lambda}$ defined in the previous section:

$$
\frac{d V_{n}(t)}{d t}=A_{n} V_{n}(t)+f_{n}\left(V_{n}(t)+Z_{t}^{\lambda}, t\right)+\lambda \pi_{n}\left(Z_{t}^{\lambda}\right), \quad V_{n}(0)=\pi_{n} V(0) .
$$

This is a random differential equation. For each $n \in \mathbb{N}$ and $\lambda>0$, as a stochastic equation, it has a unique global continuous $\mathcal{F}_{t}$-adapted solution $V_{n}$ (a strong solution, in the stochastic sense). Indeed, given any continuous path of $Z_{\text {. }}^{\lambda}$, this follows from the local Lipschitz property of each $f^{i}$, and assumption (2.3). (See e.g., [19, Theorem 3.1.1].) Whence a unique global solution is established for $P$-a.e. $\omega \in \Omega$ (those for which $Z^{\lambda}$. $(\omega)$ is continuous) and it is an adapted process, by uniqueness.

Notice that $Z_{t}^{\lambda} \in E$ for $d t$-a.e. $t \in[0, T]$ and $V_{n}(t) \in E \cap V$ (because $\pi_{n}(H) \subset$ $E \cap V$ ) for every $t \in[0, T]$, with probability one. Thus we may apply the inequalities of our assumptions with $z=Z_{t}^{\lambda}$ and $v=V_{n}(t)$.

Using the notations of inner product and norm of $H$ also in $H_{n}$, from assumption (2.3) we have

$$
\begin{aligned}
\frac{1}{2} \frac{d\left\|V_{n}\right\|_{H}^{2}}{d t}-\left\langle A_{n} V_{n}, V_{n}\right\rangle_{H} \leq & \left|\left\langle f_{n}\left(V_{n}+Z^{\lambda}, t\right), V_{n}\right\rangle_{H}\right|+\lambda\left\|Z^{\lambda}\right\|_{H}\left\|V_{n}\right\|_{H} \\
\leq & \eta\left\langle A_{n} V_{n}, V_{n}\right\rangle_{H}+C\left\|V_{n}\right\|_{H}^{2}\left(\left\|Z^{\lambda}\right\|_{E}^{2}+1\right) \\
& +C\left\|Z^{\lambda}\right\|_{E}^{k_{0}}+C+\lambda^{2}\left\|Z^{\lambda}\right\|_{H}^{2}+\left\|V_{n}\right\|_{H}^{2} .
\end{aligned}
$$

Just in order to unify some expressions, and without restriction, let us assume from now on that $k_{0} \geq 2$; otherwise it is only a matter of keeping explicitly the term $\left\|Z^{\lambda}\right\|_{H}^{2}$. With possibly changing constants $C$, we have

$$
\frac{d\left\|V_{n}\right\|_{H}^{2}}{d t}-\left\langle A_{n} V_{n}, V_{n}\right\rangle_{H} \leq C\left\|V_{n}\right\|_{H}^{2}\left(\left\|Z^{\lambda}\right\|_{E}^{2}+1\right)+\left(C+\lambda^{2}\right)\left\|Z^{\lambda}\right\|_{E}^{k_{0}}+C .
$$


By Gronwall's lemma we get (using also $\left\|V_{n}(0)\right\|_{H}^{2} \leq\|V(0)\|_{H}^{2}$ )

$$
\left\|V_{n}(t)\right\|_{H}^{2} \leq e^{I^{\lambda}(0, t)}\|V(0)\|_{H}^{2}+\int_{0}^{t} e^{I^{\lambda}(s, t)}\left(\left(C+\lambda^{2}\right)\left\|Z_{s}^{\lambda}\right\|_{E}^{k_{0}}+C\right) d s,
$$

where

$$
I^{\lambda}(s, t)=\bar{C} \int_{s}^{t}\left(\left\|Z_{r}^{\lambda}\right\|_{E}^{2}+1\right) d r
$$

and thus

$$
\left\|V_{n}(t)\right\|_{H}^{2} \leq e^{I^{\lambda}(0, T)}\left[\|V(0)\|_{H}^{2}+\int_{0}^{T}\left(\left(C+\lambda^{2}\right)\left\|Z_{s}^{\lambda}\right\|_{E}^{k_{0}}+C\right) d s\right] .
$$

We have denoted the constant in $I(s, t)$ by $\bar{C}$ to emphasize that it is not a generic constant, but the one obtained so far in that estimate; it does not depend on $\lambda$, neither on $n$ nor on $\omega$.

Notice that this inequality gives us

$$
\left\|V_{n}(t)\right\|_{H} \leq e^{\frac{1}{2} I^{\lambda}(0, T)}\left[\|V(0)\|_{H}+\left(\int_{0}^{T}\left(\left(C+\lambda^{2}\right)\left\|Z_{s}^{\lambda}\right\|_{E}^{k_{0}}+C\right) d s\right)^{1 / 2}\right]
$$

so we are not limited to work in the sequel with powers of $\left\|V_{n}(t)\right\|_{H}$ greater than 2 .

Let $p_{1}>p_{0}$ be the value given in the assumptions of the theorem.

Lemma 13 For every $p \in\left(p_{0}, p_{1}\right)$, there exists $\lambda_{p} \geq 0$ and $C_{p}>0$ such that, using the process $Z^{\lambda_{p}}$ in the previous construction, we have

$$
E\left[\sup _{t \in[0, T]}\left\|V_{n}(t)\right\|_{H}^{p}\right] \leq C_{p}
$$

for every $n \in \mathbb{N}$.

Proof From inequality (4.3) we deduce

$$
\left\|V_{n}(t)\right\|_{H}^{p} \leq C_{p, T} e^{\frac{p}{2} I^{\lambda}(0, T)}\left[\|V(0)\|_{H}^{p}+\left(\int_{0}^{T}\left(\left(C+\lambda^{2}\right)\left\|Z_{s}^{\lambda}\right\|_{E}^{k_{0}}+C\right) d s\right)^{p / 2}\right],
$$

hence, for every $q, q^{\prime} \in(1, \infty)$ such that $\frac{1}{q}+\frac{1}{q^{\prime}}=1$,

$$
\begin{aligned}
& E\left[\sup _{t \in[0, T]}\left\|V_{n}(t)\right\|_{H}^{p}\right] \\
& \leq C_{p, q, T} E\left[e^{\frac{p q}{2} I^{\lambda}(0, T)}\right]^{1 / q} E\left[\|V(0)\|^{p q^{\prime}}\right]^{1 / q^{\prime}} \\
& \quad+C_{p, q, T} E\left[e^{\frac{p q}{2} I^{\lambda}(0, T)}\right]^{1 / q} E\left[\left(\int_{0}^{T}\left(\left(C+\lambda^{2}\right)\left\|Z_{s}^{\lambda}\right\|_{E}^{k_{0}}+C\right) d s\right)^{p q^{\prime} / 2}\right]^{1 / q^{\prime}} .
\end{aligned}
$$


Choose $q^{\prime}$ such that $p q^{\prime}=p_{1}$ : this gives us $E\left[\|V(0)\|_{H}^{p q^{\prime}}\right]<\infty$. Choose $\lambda$, depending on $p, q, \bar{C}$, such that $E\left[e^{\frac{p q}{2} I^{\lambda}(0, T)}\right]<\infty$ : this is possible because of Proposition 12. Finally, for those values of the parameters, the last expected value of the last inequality is finite because $Z_{\text {. }}^{\lambda}$ is a Gaussian variable in $L^{k_{0}}(0, T ; E)$, see assumption (2.5). The proof is complete.

\subsection{An Additional Estimate}

Inequality (4.2) also gives us

$$
\begin{aligned}
& -\int_{0}^{T}\left\langle A_{n} V_{n}, V_{n}\right\rangle_{H} d s \leq\left\|V_{n}(0)\right\|_{H}^{2}+C \int_{0}^{T}\left\|V_{n}\right\|_{H}^{2}\left(\left\|Z^{\lambda}\right\|_{E}^{2}+1\right) d s \\
& +\left(C+\lambda^{2}\right) \int_{0}^{T}\left\|Z^{\lambda}\right\|_{E}^{k_{0}} d s+C T
\end{aligned}
$$

Recalling the definition of the Hilbert space $V$ given in the introduction, we have proved our additional estimate:

$$
\begin{gathered}
\int_{0}^{T}\left\|V_{n}(s)\right\|_{V}^{2} d s \leq \\
+\left(C+V_{n}(0)\left\|_{H}^{2}+C \int_{0}^{T}\right\| \int_{0}^{T}\left\|V_{n}\right\|_{H}^{2} \|_{E}^{k_{0}} d s+C T\right. \\
+\left(\left\|Z^{\lambda}\right\|_{E}^{2}+1\right) d s
\end{gathered}
$$

In fact, for our later purposes we may simplify it as follows:

$$
\int_{0}^{T}\left\|V_{n}(s)\right\|_{V}^{2} d s \leq C \sup _{[0, T]}\left\|V_{n}\right\|_{H}^{4}+\left(\int_{0}^{T}\left\|Z^{\lambda}\right\|_{E}^{k_{0} \vee 2} d s\right)^{2}+C
$$

for all $n \in \mathbb{N}$ with a new constant $C$, depending also on $\lambda$ and $T$, but independent of $n$ and $\omega$.

\subsection{Approximating Fokker-Planck Equation}

Given $n \in \mathbb{N}$ define $D\left(\widetilde{L}_{n}\right)$ to be the span of all functions $\widetilde{u}_{n}: H_{n} \times H \times[0, T] \rightarrow \mathbb{R}$ such that

$$
\tilde{u}_{n}(v, z, t)=\tilde{u}_{n, N}\left(v,\left\langle e_{1}, z\right\rangle, \ldots,\left\langle e_{N}, z\right\rangle, t\right),
$$

for some $\tilde{u}_{n, N} \in C_{b}^{2,1}\left(H_{n} \times H_{N} \times[0, T]\right)\left(\right.$ i.e., $C^{2}$ on $H_{n} \times H_{N}$ and $C^{1}$ on $\left.[0, T]\right)$ and $\tilde{u}_{n}(v, z, T)=0$. Consider on $H_{n} \times H$ the Fokker-Planck equation

$$
\begin{aligned}
& \int_{0}^{T} \int_{H_{n} \times H}\left(\widetilde{L}_{n} \widetilde{u}_{n}\right)(v, z, t) \tilde{\mu}_{t}^{n}(d v, d z) d t \\
& \quad+\int_{H_{n} \times H} \widetilde{u}_{n}(v, z, 0) \tilde{\mu}_{0}^{n}(d v, d z)=0, \quad \forall \widetilde{u}_{n} \in D\left(\widetilde{L}_{n}\right),
\end{aligned}
$$


where for $(v, z, t) \in H_{n} \times H \times[0, T]$

$$
\begin{aligned}
\left(\widetilde{L}_{n} \widetilde{u}_{n}\right)(v, z, t):= & \frac{\partial \widetilde{u}_{n}}{\partial t}(v, z, t)+\sum_{i=1}^{\infty}\left(\left(a^{i} \partial_{z_{i}}^{2}-\left(\alpha_{i}^{2}+\lambda\right) z_{i} \partial_{z_{i}}\right) \widetilde{u}_{n}\right)(v, z, t) \\
& +\sum_{i=1}^{n}\left(-\alpha_{i}^{2} v_{i}+f^{i}(v+z, t)+\lambda z_{i}\right)\left(\partial_{v_{i}} \widetilde{u}_{n}\right)(v, z, t) .
\end{aligned}
$$

The initial measure $\widetilde{\mu}_{0}^{n}$ is, by definition, the projection on $H_{n} \times H$ of the given initial datum $\tilde{\mu}_{0}$.

Let $\left(V_{n}, Z^{\lambda}\right)$ be the process constructed in the previous section. For $n \in \mathbb{N}, t \in$ $[0, T]$, define $\widetilde{\mu_{t}^{n}}(d v, d z)$ to be the law of $\left(V_{n}(t), Z_{t}^{\lambda}\right)$. Then clearly $\widetilde{\mu_{t}^{n}}(d v, d z) d t$ solves $\left(\widetilde{F P E^{n}}\right)$ by Itô's formula. Replacing $\widetilde{u}_{n}(v, z, t)$ in $\left(\widetilde{F P E^{n}}\right)$ by $\varphi(t) \widetilde{u}_{n}(v, z, t)$ for $\varphi \in C_{0}([0, T))$ we easily see that $\left(\widetilde{F P E^{n}}\right)$ is equivalent to

$$
\begin{aligned}
& \int_{H_{n} \times H} u(v, z, t) \tilde{\mu}_{t}^{n}(d v, d z) \\
& =\int_{H_{n} \times H} u(v, z, 0) \tilde{\mu}_{0}^{n}(d v, d z) \\
& \quad+\int_{0}^{t} \int_{H_{n} \times H} \widetilde{L}_{n} u(v, z, s) \tilde{\mu}_{s}^{n}(d v, d z) d s, \quad \forall t \in[0, T], u \in D\left(\widetilde{L}^{n}\right) .
\end{aligned}
$$

(See [7, Remark 1.2] for details.) Hence an easy consideration shows that the above equation also holds for all $\varphi \in \mathcal{F} C_{b}^{2}$, i.e., all functions $\varphi: H \times H \rightarrow \mathbb{R}$ of the form

$$
\varphi(v, z)=\varphi_{N}\left(\left\langle e_{1}, v\right\rangle, \ldots,\left\langle e_{N}, v\right\rangle,\left\langle e_{1}, z\right\rangle, \ldots,\left\langle e_{N}, z\right\rangle\right),
$$

where $N \in \mathbb{N}, \varphi_{N} \in C_{b}^{2}\left(\mathbb{R}^{N} \times \mathbb{R}^{N}\right)$. Hence it follows from Remark 6 and Lemma 13 that for every $\varphi \in \mathcal{F} C_{b}^{2}$ the $\mathbb{R}$-valued maps

$$
t \rightarrow \tilde{\mu}_{t}^{n}(\varphi):=\int_{H_{n} \times H} \varphi(v, z) \tilde{\mu}_{t}^{n}(d v, d z), \quad n \in \mathbb{N},
$$

are equicontinuous on $[0, T]$.

\subsection{Passage to the Limit}

Step 1. Convergence of a subsequence of $\widetilde{\mu}_{t}^{n}, n \in \mathbb{N}$, for all $t \in[0, T]$.

First we extend $\tilde{\mu}_{t}^{n}$ from a measure on $H_{n} \times H$ to a measure on $H \times H$ as follows. Let $H_{n}^{\perp}$ be the orthogonal complement of $H_{n}$ in $H, \delta_{0}$ the Dirac measure on $H_{n}^{\perp}$ with mass at $0 \in H_{n}^{\perp}$ and $\Lambda: H_{n} \times H_{n}^{\perp} \times H \rightarrow H \times H$ defined by

$$
\Lambda\left(\left(v_{n}, v_{n}^{\perp}, z\right)\right)=\left(v_{n}+v_{n}^{\perp}, z\right), \quad v_{n} \in H_{n}, v_{n}^{\perp} \in H_{n}^{\perp}, z \in H .
$$

Then the image under $\Lambda$ of the measure $\widetilde{\mu}_{t}^{n}\left(d v_{n}, d z\right) \otimes \delta_{0}\left(d v_{n}^{\perp}\right)$ extends $\widetilde{\mu}_{t}^{n}$ to $H \times H$. Let us also denote this extension by $\tilde{\mu}_{t}^{n}$. Then we have for any integrable function 
$g: H \times H \rightarrow \mathbb{R}$

$$
\begin{aligned}
& \int_{H \times H} g(v, z) \tilde{\mu}_{t}^{n}(d v, d z) \\
& =\int_{H_{n} \times H_{n}^{\perp} \times H} g\left(v_{n}+v_{n}^{\perp}, z\right) \tilde{\mu}_{t}^{n}\left(d v_{n}, d z\right) \otimes \delta_{0}\left(d v_{n}^{\perp}\right) \\
& =\int_{H_{n} \times H} g\left(v_{n}, z\right) \tilde{\mu}_{t}^{n}\left(d v_{n}, d z\right) \otimes \delta_{0}\left(d v_{n}^{\perp}\right) \\
& =\int_{H \times H} g\left(\pi_{n}(v), z\right) \tilde{\mu}_{t}^{n}(d v, d z) .
\end{aligned}
$$

Furthermore, by Remark 7 and Lemma 13 for each $t \in[0, T]$

$$
\sup _{n \in \mathbb{N}} \int_{H \times H}\left(\|v\|_{H}^{p}+\|z\|_{H}^{2}\right) \widetilde{\mu}_{t}^{n}(d v, d z)<+\infty
$$

for any $p$ given in Lemma 13. Closed balls in $H \times H$ are compact and metrizable with respect to the weak topology $\tau_{w}$. Hence by [3, Theorem 8.6.7] and a diagonal argument we can find a subsequence $\tilde{\mu}_{t}^{n_{k}}, k \in \mathbb{N}$, such that $\left(\tilde{\mu}_{t}^{n_{k}}\right)$ converges $\tau_{w^{-}}$ weakly to a probability measure $\tilde{\mu}_{t}$ on $H \times H$ as $k \rightarrow \infty$ for all $t \in[0, T] \cap \mathbb{Q}$. Now let $t \in[0, T] \backslash \mathbb{Q}$. We claim that also for such $t$ the sequence $\left(\tilde{\mu}_{t}^{n_{k}}\right)$ converges $\tau_{w}$-weakly to some probability measure $\tilde{\mu}_{t}$ on $H \times H$. Since by (4.7) also $\left(\tilde{\mu}_{t}^{n_{k}}\right)$ has $\tau_{w}$-convergent subsequences, we only have to identify the limit points. So, let $n_{k_{l}}, l \in \mathbb{N}$, be a subsequence such that $\left(\tilde{\mu}_{t}^{n_{k_{l}}}\right) \tau_{w}$-weakly converges to some probability measure $\widetilde{v}_{t}$ on $H \times H$ as $l \rightarrow \infty$. Then by the equicontinuity proved in Sect. 4.4 we have for all $\varphi \in \mathcal{F} C_{b}^{2}$ (which are all weakly continuous)

$$
\begin{aligned}
\int_{H \times H} \varphi d \widetilde{v}_{t} & =\lim _{l \rightarrow \infty} \int_{H \times H} \varphi d \tilde{\mu}_{t}^{n_{k_{l}}} \\
& =\lim _{l \rightarrow \infty} \lim _{s \rightarrow t, s \in \mathbb{Q}} \int_{H \times H} \varphi d \widetilde{\mu}_{s}^{n_{k_{l}}}=\lim _{s \rightarrow t, s \in \mathbb{Q}} \int_{H \times H} \varphi d \tilde{\mu}_{s} .
\end{aligned}
$$

Since $\mathcal{F} C_{b}^{2}$ is a measure separating class, this proves our claim.

Step 2. Convergence of a subsequence of $\widetilde{\mu}_{t}^{n}(d v, d z) d t$.

Let $\left(\gamma_{i}\right) \subset[1, \infty)$ such that

$$
\gamma_{i} \uparrow \infty, \quad \sum_{i=1}^{\infty} \gamma_{i} \frac{a_{i}}{\alpha_{i}^{2}}<\infty .
$$


Define the operator $\Gamma$ on $H$ by

$$
\left\{\begin{array}{l}
\Gamma x:=\sum_{i=1}^{\infty} \gamma_{i}^{1 / 2}\left\langle x, e_{i}\right\rangle e_{i}, \\
D(\Gamma):=\left\{x \in H: \sum_{i=1}^{\infty} \gamma_{i}\left\langle x, e_{i}\right\rangle^{2}<\infty\right\} .
\end{array}\right.
$$

Then $\Gamma$ has compact level sets and furthermore

$$
\begin{aligned}
\mathbb{E}\left[\left\|\Gamma\left(Z_{t}^{\lambda}\right)\right\|_{H}^{2}\right] & =\sum_{i=1}^{\infty} \gamma_{i} a^{i} \int_{0}^{t} e^{-2(t-s)\left(\alpha_{i}^{2}+\lambda\right)} d s \\
& =\frac{1}{2} \sum_{i=1}^{\infty} \gamma_{i} \frac{a^{i}}{\alpha_{i}^{2}+\lambda}\left(1-e^{-2 t\left(\alpha_{i}^{2}+\lambda\right)}\right) \\
& \leq \frac{1}{2} \sum_{i=1}^{\infty} \gamma_{i} \frac{a^{i}}{\alpha_{i}^{2}}=: C_{\gamma}<\infty .
\end{aligned}
$$

Hence it follows from (2.5), (4.5) and Lemma 13

$$
\sup _{n \in \mathbb{N}} \int_{0}^{T} \int_{H \times H}\left(\|v\|_{V}^{2}+\|\Gamma z\|_{H}^{2}\right) \tilde{\mu}_{t}^{n}(d v, d z) d t<\infty .
$$

But the function $(v, z) \rightarrow\|v\|_{V}^{2}+\|\Gamma v\|_{H}^{2}$ has compact level sets on $H \times H$, hence (selecting a subsequence if necessary) $\widetilde{\mu}_{t}^{n_{k}}(d v, d z) d t$, where $n_{k}, k \in \mathbb{N}$, is as in Step 1 , weakly converges to a finite measure $\tilde{\mu}(d t, d v, d z)$ on $[0, T] \times H \times H$ as $k \rightarrow \infty$. But for $u \in D(\widetilde{L})$ we then have by Lebesgue's dominated convergence theorem

$$
\begin{aligned}
\int_{0}^{T} & \int_{H \times H} u(v, z, t) \tilde{\mu}_{t}(d v, d z) d t \\
\quad= & \lim _{k \rightarrow \infty} \int_{0}^{T} \int_{H \times H} u(v, z, t) \tilde{\mu}_{t}^{n_{k}}(d v, d z) d t \\
& =\int_{0}^{T} \int_{H \times H} u(v, z, t) \tilde{\mu}(d t, d v, d z) .
\end{aligned}
$$

Since $D(\widetilde{L})$ is a measure separating class, it follows that $\tilde{\mu}(d t, d v, d z)=\tilde{\mu}_{t}(d v$, $d z) d t$. So $\widetilde{\mu}_{t}^{n_{k}}(d v, d z) d t \rightarrow \widetilde{\mu}_{t}(d v, d z) d t$ weakly on $H \times H \times[0, T]$ as $k \rightarrow \infty$.

Step 3. Passage to the limit.

Just to simplify notations, assume that the whole sequence $\left(\widetilde{\mu}_{t}^{n}(d v, d z) d t\right)_{n \in \mathbb{N}}$ weakly converges to $\tilde{\mu}_{t}(d v, d z) d t$ on $[0, T] \times H \times H$. We have to prove that 
$\tilde{\mu}_{t}(d v, d z) d t$ is a solution of $(\widetilde{F P E})$. Since we have $\widetilde{\mu}_{0}^{n} \rightarrow \widetilde{\mu}_{0}$ weakly on $H \times H$ we only need to prove that

$$
\begin{gathered}
\lim _{n \rightarrow \infty} \int_{0}^{T} \int_{H \times H}(\widetilde{L} \tilde{u})(v, z, t) \widetilde{\mu}_{t}^{n}(d v, d z) d t \\
\quad=\int_{0}^{T} \int_{H \times H}(\widetilde{L} \widetilde{u})(v, z, t) \widetilde{\mu}_{t}(d v, d z) d t
\end{gathered}
$$

for all $\tilde{u} \in D(\widetilde{L})$. Below we fix such a $\tilde{u}$. Let $m \in \mathbb{N}$ such that

$$
\widetilde{u}(v, z, t)=\widetilde{u}\left(\pi_{m} v, \pi_{m} z, t\right)
$$

and let $n \geq m$. By (4.6) the above equation follows from

$$
\begin{gathered}
\lim _{n \rightarrow \infty, n \geq m} \int_{0}^{T} \int_{H \times H} \psi(v, z, t) \tilde{\mu}_{t}^{n}(d v, d z) d t \\
=\int_{0}^{T} \int_{H \times H} \psi(v, z, t) \tilde{\mu}_{t}(d v, d z) d t,
\end{gathered}
$$

where

$$
\begin{aligned}
\psi(v, z, t)= & -\sum_{i=1}^{m}\left(\alpha_{i}^{2}+\lambda\right) z_{i} \partial_{z_{i}} \widetilde{u}\left(\pi_{m} v, \pi_{m} z, t\right) \\
& +\sum_{i=1}^{m}\left(-\alpha_{i}^{2} v_{i}+\lambda z_{i}\right) \partial_{v_{i}} \widetilde{u}\left(\pi_{m} v, \pi_{m} z, t\right) \\
& +\sum_{i=1}^{m} f^{i}(v+z, t) \partial_{v_{i}} \widetilde{u}(v, z, t) .
\end{aligned}
$$

Note that $\psi$ is a continuous function, but not bounded. So, we cannot pass to the limit in (4.8) just by the weak convergence of $\widetilde{\mu}_{t}^{n} d t$ to $\tilde{\mu}_{t} d t$ on $H \times H \times[0, T]$ proved in Step 2 above. But we can argue similarly as in the proof of Vitali's theorem. Let us give the details.

By assumption (2.2) we have

$$
|\psi(v, z, t)| \leq c\left(1+\|v\|_{H}^{p_{0}}+\|z\|_{H}^{p_{0}}\right) .
$$

For $R \in(0, \infty)$ we define

$$
\psi_{R}:=\psi \wedge R \vee(-R),
$$

then

$$
\psi=\psi_{R}+\left(\psi-\psi_{R}\right)
$$


where

$$
\left|\psi-\psi_{R}\right| \leq \mathbb{1}_{\{|\psi| \geq R\}} 2|\psi| .
$$

Hence by Step 2 it suffices to show that

$$
\limsup _{R \rightarrow \infty} \sup _{n \in \mathbb{N}} \int_{0}^{T} \int_{H \times H} \mathbb{1}_{\{|\psi| \geq R\}}|\psi| d \tilde{\mu}_{t}^{n} d t=0
$$

and

$$
\limsup _{R \rightarrow \infty} \int_{0}^{T} \int_{H \times H} \mathbb{1}_{\{|\psi| \geq R\}}|\psi| d \tilde{\mu}_{t} d t=0 .
$$

But we have by the Hölder inequality for every $\delta \in(0, \infty)$

$$
\begin{aligned}
\int_{0}^{T} \int_{H \times H} \mathbb{1}_{\{|\psi| \geq R\}}|\psi| d \tilde{\mu}_{t}^{n} d t \leq & \left(\int_{0}^{T} \int_{H \times H}|\psi|^{1+\delta} d \tilde{\mu}_{t}^{n} d t\right)^{\frac{1}{1+\delta}} \\
& \times\left(\frac{1}{R} \int_{0}^{T} \int_{H \times H}|\psi| d \tilde{\mu}_{t}^{n} d t\right)^{\frac{\delta}{1+\delta}} .
\end{aligned}
$$

By Lemma 13 and (4.9) this implies (4.10). Note that by Step 2 and Lemma 13 it follows by lower continuity that

$$
\int_{0}^{T} \int_{H \times H}\left(\|v\|_{H}^{p}+\|z\|_{H}^{p}\right) \tilde{\mu}_{t}(d v, d z) d t<\infty,
$$

for all $p \in\left(p_{0}, p_{1}\right)$. Hence (4.11) follows similarly (even easier) as (4.10) above.

\section{Examples}

\subsection{Measurable Linear Growth Drift}

Let $f: H \times[0, T] \rightarrow H$ be a measurable map such that

$$
\|f(x, t)\|_{H} \leq C\left(1+\|x\|_{H}\right), \quad t \in[0, T], x \in H
$$

for some constant $C>0$. Denote by $f^{i}(t, x)$ its components. Assume that (2.1) holds. Then, with $E=H$, also the other assumptions hold. The proof of assumptions (2.2) and (2.5) is elementary. We have

$$
\langle f(v+z, t), v\rangle_{H} \leq C\left(1+\|v+z\|_{H}\right)\|v\|_{H} \leq C^{\prime}\|v\|_{H}^{2}+C^{\prime}\|z\|_{H}^{2}+C^{\prime \prime}
$$

which implies (2.3). Finally,

$$
E\left[\left\|Z_{t}^{\lambda}\right\|_{H}^{2}\right]=\sum_{i=1}^{\infty}\left(1-e^{-2 t\left(\alpha_{i}^{2}+\lambda\right)}\right) \frac{a^{i}}{2\left(\alpha_{i}^{2}+\lambda\right)} \leq \sum_{i=1}^{\infty} \frac{a^{i}}{2\left(\alpha_{i}^{2}+\lambda\right)} .
$$


Hence

$$
\begin{aligned}
P\left(\int_{0}^{T}\left\|Z_{t}^{\lambda}\right\|_{E}^{2} d t>r^{2}\right) & \leq r^{-2} E\left[\int_{0}^{T}\left\|Z_{t}^{\lambda}\right\|_{H}^{2} d t\right]=r^{-2} \int_{0}^{T} E\left[\left\|Z_{t}^{\lambda}\right\|_{H}^{2}\right] d t \\
& =\frac{1}{2} r^{-2} T \sum_{i=1}^{\infty} \frac{a^{i}}{\alpha_{i}^{2}+\lambda}
\end{aligned}
$$

which implies (2.6). Note that (2.4) follows from (2.1). Hence Theorem 4 holds.

Remark 14 Similarly as in [20] we can also treat stochastic partial differential equations on $H=L^{2}(0,1)$ whose drift is the sum of the Dirichlet Laplacian, a reactiondiffusion and a Burgers type part. However, in contrast to [20] (and also [5, 7]) we need to assume that the reaction part is of at most quadratic growth. The details are straightforward.

\subsection{Navier-Stokes Equations}

Consider the stochastic Navier-Stokes equations

$$
\begin{aligned}
d u+(u \cdot \nabla u+\nabla p) d t & =v \Delta u d t+\sum_{i=1}^{\infty} \sqrt{a^{i}} e_{i} d \beta_{i}(s), \\
\operatorname{div} u & =0 \\
\left.u\right|_{t=0} & =u_{0}
\end{aligned}
$$

on the torus $D=[0,2 \pi]^{d}, d=2,3$, with periodic boundary conditions.

We introduce the Hilbert space $H$ defined as the closure in $L^{2}\left(D, \mathbb{R}^{d}\right)$ of the set of all $\varphi \in C^{\infty}\left(D, \mathbb{R}^{d}\right)$ which satisfy the periodic boundary conditions and $\operatorname{div} \varphi=0 ; H$ is a closed strict subspace of $L^{2}\left(D, \mathbb{R}^{d}\right)$ and we shall denote the orthogonal projection from $L^{2}\left(D, \mathbb{R}^{d}\right)$ to $H$ by $P_{H}$. We assume $u_{0} \in H$. We introduce also the Hilbert space $V$ of all periodic $\varphi \in H^{1}\left(D, \mathbb{R}^{d}\right)$ such that $\operatorname{div} \varphi=0 ;$ and $D(A)=H^{2}\left(D, \mathbb{R}^{d}\right) \cap V$. Then we introduce the so called Stokes operator $A: D(A) \subset H \rightarrow H$ defined as $A \varphi=P_{H}(\nu \Delta \varphi)$ (in fact, in the case of periodic boundary conditions, one can show that $A \varphi=v \Delta \varphi)$. Since $A^{-1}$ is compact, there exists a complete orthonormal system $\left\{e_{i}\right\}$ of eigenvectors of $A$, with eigenvalues $\left\{-\alpha_{i}^{2}\right\}$, that we order such that $0<\alpha_{1}^{2} \leq$ $\alpha_{2}^{2} \leq \cdots$. One can show that, with these new concepts and notations, the space $V$ defined in Sect. 2 and the space $V$ defined here coincide.

Let $B(.,):. D(A) \times V \rightarrow H$ be defined as

$$
B(\varphi, \psi)=-P_{H}(\varphi \cdot \nabla \psi) .
$$

The expression $\int_{D} B(\varphi, \psi)(x) \theta(x) d x, \varphi \in D(A), \psi \in V, \theta \in H$, extends to $\varphi, \psi, \theta \in V$, and several other classes of functions. For smooth fields $\varphi, \psi, \theta \in H$ we have

$$
\langle B(\varphi, \psi), \theta\rangle=-\langle B(\varphi, \theta), \psi\rangle
$$


by a simple integration by parts, and this identity extends by density to several spaces of weaker fields.

Using the previous set-up we may formally write the stochastic Navier-Stokes equations in abstract form (the pressure disappears since $P_{H}(\nabla p)=0$ ):

$$
d u=(A u+B(u, u)) d t+\sum_{i=1}^{\infty} \sqrt{a^{i}} e_{i} d \beta_{i}(s) .
$$

See [15] for a review on the 3D stochastic Navier-Stokes equations and further details on the set-up.

To connect this equation with the abstract framework of this paper we consider the space $E=C_{\text {per }}\left(D ; \mathbb{R}^{d}\right) \cap H$, where $C_{\text {per }}\left(D ; \mathbb{R}^{d}\right)$ is the space of periodic continuous vector fields on $D$, introduce the functions $f^{i}:[0, T] \times H \rightarrow \mathbb{R}$ defined as

$$
f^{i}(t, x)=-\left\langle B\left(x, e_{i}\right), x\right\rangle=\int_{D}(x(\xi) \cdot \nabla) e_{i}(\xi) \cdot x(\xi) d \xi
$$

and consider the sequences $\left\{\alpha_{i}^{2}\right\}$ and $\left\{a_{i}\right\}$ above. As remarked above, since $\int_{D} B(\varphi, \psi)(x) \theta(x) d x$ extends to $\varphi, \psi, \theta \in V$, there exists $f(t, x)$ with values in $V^{\prime}$ such that $f^{i}(x, t)=\left\langle f(x, t), e_{i}\right\rangle$; it is given by $B(x, x)$, when $x \in D(A)$.

We assume that $a_{i}$ has the form

$$
a^{i}=\alpha_{i}^{-\varepsilon}
$$

for some $\varepsilon$ such that

$$
\begin{array}{ll}
\varepsilon>0, & \text { for } d=2, \\
\varepsilon>1, & \text { for } d=3 .
\end{array}
$$

This guarantees assumption (2.1). Indeed, on the torus $D$, the family of eigenvectors $\left\{e_{i}\right\}_{i \in \mathbb{N}}$ of $A$ can be written (see [21]) in the form $\left\{e_{\alpha, k}\right\}$ with $k \in \mathbb{Z}_{*}^{d}=\mathbb{Z}^{d} \backslash\{0\}$ and $\alpha$ which varies in the finite set $\{1, \ldots, d-1\}$ and their associated eigenvalues, indexed in the form $\left\{\alpha_{k}^{2}\right\}_{k \in \mathbb{Z}_{*}^{d}}$ (for each $k \in \mathbb{Z}_{*}^{d}$ the eigenvalue $\alpha_{k}^{2}$ has multiplicity $d-1$ ), are given by $\alpha_{k}^{2}=\|k\|^{2}$. If we use the complex valued notation, one has $e_{\alpha, k}(\xi)=$ $c_{\alpha, k} e^{i k \cdot \xi}$ where, for each $k$, the set of vectors $\left\{c_{\alpha}\right\}_{\alpha \in\{1, \ldots, d-1\}}$ is an orthonormal basis of the space in $\mathbb{R}^{d}$ orthogonal to $k$.

Hence we may rewrite $\sum_{i=1}^{\infty} \frac{a^{i}}{\alpha_{i}^{2}}$ as

$$
\sum_{k \in \mathbb{Z}_{*}^{d}}\|k\|^{-2-\varepsilon}
$$

and this series converge in $d=2$ for every $\varepsilon>0$, and in $d=3$ for every $\varepsilon>1$.

We claim that under these conditions all the assumptions of the paper are verified and thus Theorem 4 holds. Let us check the assumptions.

The eigenvectors $e_{i}$ and their derivatives are bounded functions and thus assumption (2.2) holds with $p_{0}=2$. 
We have, for smooth fields $v, z$,

$$
\begin{aligned}
\langle f(v+z, t), v\rangle & =\int_{D}((v+z) \cdot \nabla)(v+z) \cdot v d \xi=\int_{D}((v+z) \cdot \nabla) z \cdot v d \xi \\
& =-\int_{D}((v+z) \cdot \nabla) v \cdot z d \xi \leq \eta\|v\|_{H^{1}(D)}^{2}+C_{\eta}\||z||v+z|\|_{L^{2}(D)}^{2} \\
& \leq \eta\|v\|_{H^{1}(D)}^{2}+2 C_{\eta}\|z\|_{L^{\infty}(D)}^{2}\|z\|_{L^{2}(D)}^{2}+2 C_{\eta}\|z\|_{L^{\infty}(D)}^{2}\|v\|_{L^{2}(D)}^{2}
\end{aligned}
$$

and the inequality extends to all $z \in E, v \in E \cap V$. Thus (2.3) holds true, with $k_{0}=4$.

Finally, assumptions (2.5) and (2.6) are true under the condition imposed above on $\left\{\alpha_{i}^{2}\right\}$ and $\left\{a^{i}\right\}$. To show this, we have to use the theory of Sect. 5.5.1 of [12] and the explicit form of the eigenfunctions $e_{i}$ of the Stokes operator $A$. Since we need the bounds of this reference with a precise control of the constants, we have repeated some of the computations in the next lemma.

Lemma 15 Assume $a^{i}=\alpha_{i}^{-\varepsilon}$ with $\varepsilon$ as above. Then the random field

$$
Z_{t}^{\lambda}(\xi)=\sum_{i=1}^{\infty} \int_{0}^{t} e^{-(t-s)\left(\alpha_{i}^{2}+\lambda\right)} \sqrt{a^{i}} d \beta_{i}(s) e_{i}(\xi)
$$

has a continuous modification in $(t, \xi)$ and satisfies assumptions (2.5) and (2.6).

Proof The eigenfunctions $e_{i}$ have the properties $e_{i} \in C^{1}\left(D ; \mathbb{R}^{d}\right),\left|e_{i}(\xi)\right| \leq C$, $\left|\nabla e_{i}(\xi)\right| \leq C \alpha_{i}$, required in Sect. 5.5.1 of [12]. We have also the other property asked in that reference, namely $\sum_{i=1}^{\infty} \frac{a^{i}}{\left(\alpha_{i}^{2}\right)^{1-\delta}}<\infty$ for some $\delta>0$, and precisely, for the sequel, we take $\delta=\frac{\varepsilon}{4}$, then

$$
\sum_{i=1}^{\infty} \frac{a^{i}}{\left(\alpha_{i}^{2}\right)^{1-\frac{\varepsilon}{4}}}<\infty
$$

Indeed, the previous series is equal to $\sum_{k \in \mathbb{Z}_{*}^{d}}\|k\|^{-2-\frac{\varepsilon}{2}}<\infty$ with the equivalent language of the indexes $k \in \mathbb{Z}_{*}^{d}$. Hence Theorem 5.20 of [12] applies and gives us the existence of a continuous modification of $Z_{t}^{\lambda}(\xi)$ in $(t, \xi)$. Let us be more precise from the quantitative viewpoint. In the sequel, we write $Z_{t}^{\lambda}(\xi)$ for each one of its $d$ components, for notational simplicity. It is sufficient to prove assumptions (2.5) and (2.6) for each component of $Z_{t}^{\lambda}(\xi)$.

Lemma 5.19 of [12] gives us the estimate

$$
E\left[\left|Z_{t}^{\lambda}(\xi)-Z_{t}^{\lambda}\left(\xi^{\prime}\right)\right|^{2}\right] \leq C_{1}(\lambda)\left|\xi-\xi^{\prime}\right|^{\frac{\varepsilon}{4}}, \quad \forall t \in[0, \infty),
$$

where

$$
C_{1}(\lambda):=C_{1} \sum_{i=1}^{\infty} \frac{a^{i} \alpha_{i}^{\frac{\varepsilon}{4}}}{\alpha_{i}^{2}+\lambda}
$$


for some constant $C_{1}>0$. Indeed

$$
\begin{aligned}
& E\left[\left|Z_{t}^{\lambda}(\xi)-Z_{t}^{\lambda}\left(\xi^{\prime}\right)\right|^{2}\right] \\
& =E\left[\left|\sum_{i=1}^{\infty} \int_{0}^{t} e^{-(t-s)\left(\alpha_{i}^{2}+\lambda\right)} \sqrt{a^{i}} d \beta_{i}(s)\left(e_{i}(\xi)-e_{i}\left(\xi^{\prime}\right)\right)\right|^{2}\right] \\
& =\sum_{i=1}^{\infty}\left|e_{i}(\xi)-e_{i}\left(\xi^{\prime}\right)\right|^{2} \int_{0}^{t} e^{-2(t-s)\left(\alpha_{i}^{2}+\lambda\right)} a^{i} d s \\
& \leq C \sum_{i=1}^{\infty} \frac{a^{i} \alpha_{i}^{\frac{\varepsilon}{4}}}{\alpha_{i}^{2}+\lambda}\left|\xi-\xi^{\prime}\right|^{\frac{\varepsilon}{4}}
\end{aligned}
$$

because

$$
\left|e_{i}(\xi)-e_{i}\left(\xi^{\prime}\right)\right| \leq C \alpha_{i}^{\frac{\varepsilon}{4}}\left|\xi-\xi^{\prime}\right|^{\frac{\varepsilon}{4}}
$$

Then

$$
E\left[\left|Z_{t}^{\lambda}(\xi)-Z_{t}^{\lambda}\left(\xi^{\prime}\right)\right|^{2 m}\right] \leq C_{m}(\lambda)\left|\xi-\xi^{\prime}\right|^{\frac{\varepsilon m}{4}}, \quad \forall t \in[0, \infty),
$$

where

$$
C_{m}(\lambda):=C_{m} C_{1}(\lambda)^{m}
$$

for some constant $C_{m}>0$. We remark also the easier estimate

$$
E\left[\left|Z_{t}^{\lambda}(\xi)\right|^{2 m}\right] \leq \widetilde{C}_{m}(\lambda):=\widetilde{C}_{m} \widetilde{C}_{1}(\lambda)^{m}, \quad \forall t \in[0, \infty),
$$

where

$$
\widetilde{C}_{1}(\lambda)=\widetilde{C}_{1} \sum_{i=1}^{\infty} \frac{a^{i}}{\alpha_{i}^{2}+\lambda}
$$

for some constant $\widetilde{C}_{1}>0$.

Given $\alpha \in(0,1)$, for the $W^{\alpha, 2 m}(D)$-norm, we have the estimate

$$
\begin{aligned}
& E\left[\int_{D}\left|Z_{t}^{\lambda}(\xi)\right|^{2 m} d \xi\right]+E\left[\int_{D} \int_{D} \frac{\left|Z_{t}^{\lambda}(\xi)-Z_{t}^{\lambda}\left(\xi^{\prime}\right)\right|^{2 m}}{\left|\xi-\xi^{\prime}\right|^{d+2 m \alpha}} d \xi d \xi^{\prime}\right] \\
& \quad \leq(2 \pi)^{d} \widetilde{C}_{m}(\lambda)+C_{m}(\lambda) \int_{D} \int_{D}\left|\xi-\xi^{\prime}\right|^{\frac{\varepsilon m}{4}-d-2 m \alpha} d \xi d \xi^{\prime} \\
& =: \widetilde{C}_{m}(\lambda)+C_{m}(\lambda) \cdot C_{m, \alpha, \varepsilon, d}
\end{aligned}
$$

and $C_{m, \alpha, \varepsilon, d}<\infty$ if $\frac{\varepsilon m}{4}-d-2 m \alpha>-d$, namely $\alpha<\frac{\varepsilon}{8}$. Choose $\alpha=\frac{\varepsilon}{10}$ in the sequel. We have $W^{\frac{\varepsilon}{10}}, 2 m(D) \subset C(D)$ for $2 m \frac{\varepsilon}{10}>d$, namely for $m>\frac{5 d}{\varepsilon}$. Choose for the sequel $m$ equal to the smallest integer such that $m>\frac{5 d}{\varepsilon}, m \geq 2 \vee k_{0}$ with $k_{0}=4$. Then

$$
E\left[\left\|Z_{t}^{\lambda}\right\|_{L^{\infty}(D)}^{2 m}\right] \leq C_{m}^{\prime}\left(\widetilde{C}_{m}(\lambda)+C_{m}(\lambda) \cdot C_{m, \alpha, \varepsilon, d}\right)
$$


for some constant $C_{m}^{\prime}>0$. This implies assumption (2.5). Finally, assumption (2.6) follows from $\lim _{\lambda \rightarrow \infty} C_{1}(\lambda)=0, \lim _{\lambda \rightarrow \infty} \widetilde{C}_{1}(\lambda)=0$ and Chebyshev inequality.

Remark 16 We think that in this case (2.6) also follows from Remark 1. But we could not find a suitable reference for the condition on $e^{t A}, t \geq 0$, in Remark 1 . Therefore, we have given a direct proof of (2.6) above.

Acknowledgements Support by the De Giorgi Centre and the DFG through SFB 701 is gratefully acknowledged. The last named author would also like to thank the Scuola Normale Superiore and the University of Pisa for the support and hospitality during several very pleasant visits during which large parts of this work were done.

\section{References}

1. Albeverio, S., Ferrario, B.: Uniqueness of solutions of the stochastic Navier-Stokes equation with invariant measure given by the enstrophy. Ann. Probab. 32(2), 1632-1649 (2004)

2. Albeverio, S., Ferrario, B.: Some methods of infinite dimensional analysis in hydrodynamics: an introduction. In: SPDE in Hydrodynamic: Recent Progress and Prospects. Lecture Notes in Math., vol. 1942, pp. 1-50. Springer, Berlin (2008)

3. Bogachev, V.I.: Measure Theory, vol. 2. Springer, Berlin (2007)

4. Bogachev, V.I., Da Prato, G., Röckner, M.: Parabolic equations for measures on infinite-dimensional spaces. Dokl. Akad. Nauk 421(4), 439-444 (2008) (in Russian); translation in Dokl. Math. 78(1), 544-549 (2008)

5. Bogachev, V.I., Da Prato, G., Röckner, M.: Existence and uniqueness of solutions for Fokker-Planck equations on Hilbert spaces. J. Evol. Equ. 10, 487-509 (2010)

6. Bogachev, V.I., Da Prato, G., Röckner, M.: Uniqueness for solutions of Fokker-Planck equations on infinite dimensional spaces. Commun. Partial Differ. Equ. 36, 925-939 (2011)

7. Bogachev, V.I., Da Prato, G., Röckner, M.: Existence results for Fokker-Planck equations in Hilbert spaces. In: Seminar on Stochastic Analysis, Random Fields and Applications VI. Progress in Probab., vol. 63, pp. 23-35 (2011)

8. Bogachev, V.I., Da Prato, G., Röckner, M., Shaposhnikov, S.: Analytic approach to infinite dimensional continuity and Fokker-Planck equations. CRC 701, Preprint (2013)

9. Crauel, H., Flandoli, F.: Attractors for random dynamical systems. Probab. Theory Relat. Fields 100(3), 365-393 (1994)

10. Da Prato, G., Debussche, A.: Two-dimensional Navier-Stokes equations driven by a space-time white noise. J. Funct. Anal. 196(1), 180-210 (2002)

11. Da Prato, G., Debussche, A.: Ergodicity for the 3D stochastic Navier-Stokes equations. J. Math. Pures Appl. (9) 82(8), 877-947 (2003)

12. Da Prato, G., Zabczyk, J.: Stochastic Equations in Infinite Dimensions. Cambridge University Press, Cambridge (1992)

13. Flandoli, F.: Dissipativity and invariant measures for stochastic Navier-Stokes equations. Nonlinear Differ. Equ. Appl. 1(4), 403-423 (1994)

14. Flandoli, F.: Irreducibility of the 3-D stochastic Navier-Stokes equation. J. Funct. Anal. 149(1), 160177 (1997)

15. Flandoli, F.: An introduction to 3D stochastic fluid dynamics. In: SPDE in Hydrodynamic: Recent Progress and Prospects. Lecture Notes in Math., vol. 1942, pp. 51-150. Springer, Berlin (2008)

16. Flandoli, F., Gatarek, D.: Martingale and stationary solutions for stochastic Navier-Stokes equations. Probab. Theory Relat. Fields 102(3), 367-391 (1995)

17. Flandoli, F., Romito, M.: Partial regularity for the stochastic Navier-Stokes equations. Trans. Am. Math. Soc. 354(6), 2207-2241 (2002)

18. Flandoli, F., Romito, M.: Markov selections for the 3D stochastic Navier-Stokes equations. Probab. Theory Relat. Fields 140(3-4), 407-458 (2008) 
19. Prêvot, C., Röckner, M.: A Concise Course on Stochastic Partial Differential Equations. Lecture Notes in Mathematics, vol. 1905. Springer, Berlin (2007)

20. Röckner, M., Zhu, R., Zhu, X.: A note on stochastic semilinear equations and their associate FokkerPlanck equations. CRC 701, Preprint (2013)

21. Temam, R.: Navier-Stokes Equations and Nonlinear Functional Analysis. CBMS-NSF Regional Conference Series in Applied Mathematics, vol. 41. SIAM, Philadelphia (1983) 